ISSN 0001-6002/2002/44/1/36-38

A cta M édica Costarricense, 02002

Colegio de M édicos y Cirujanos

\title{
Neumotórax hipertensivo por inhalación de cocaína
}

\author{
William José González Cogollo ${ }^{1}$
}

Resumen: El uso de la cocaína causa complicaciones pulmonares, cardiacas, músculo esqueléticas, obstétricas y gastrointestinales.

Se describe el caso de un varón joven quien presento barotrauma (neumotórax hipertensivo) posterior a la inhalación de cocaína.

Basados en este caso y en una revisión de la literatura medica se recomienda interrogar exhaustivamente acerca del uso de drogas en todo paciente adulto o adolescente con neumotórax "espontáneo".

Descriptores: Neumotórax, cocaína, complicaciones, abuso de drogas.

Recibido: 27 de septiembre, 2001

Aceptado para publicación: 18 de diciembre, 2001

Es una tradición milenaria de los pueblos andinos de Sudamérica mascar las hojas del arbusto de la coca ( Erythroxylon coca) para paliar los efectos del cansancio y del hambre. ${ }^{1}$

En 1860, A lbert Niemann uno de los pioneros de la química orgánica logra aislar a partir de las hojas de coca un alcaloide cristalino, incoloro y de sabor amargo al que llamó cocaína.

Básicamente hay dos formas químicas de la cocaína: una forma hidrosoluble y termolábil (clorhidrato de cocaína) que se obtiene al disolver el alcaloide en ácido clorhídrico, la cual es de alta pureza $(89 \%)^{1}$ y está disponible en nuestro medio para uso medico como anestésico local y vasoconstrictor en ciertos tipos de cirugías otorrinolaringológicas.

L a otra forma es termoresistente e insoluble en agua (pero soluble en alcohol, acetona y éter) y suele ser mezclada con sustancias tales como talco, maicena, anfetaminas, quinina, estricnina, detergentes, formol, etc. de ahí que su pureza fluctúe entre un $5 \%$ y un $50 \%$, esta forma constituye el narcótico ilegal mas popular en la actualidad y se le denomina "free-base" ${ }^{1} 0$ cocaína base, en nuestro medio se conoce

Hospital Dr. Tony Facio Castro, Limón, Costa Rica.

Servicio de Cirugía General,

Correspondencia: A partado postal 7300-601 Limón, Costa Rica Correo Electrónico: drgonzalez@ hotmail.com, telefono: 798-2259 Fax-798-0100 popularmente como "Crack", nombre que proviene del sonido que hace la mezcla al calentarse.

Como el uso de la cocaína se ha hecho mas popular en los últimos años, es de esperar que las complicaciones médicas relacionadas con su uso sean cada vez mas frecuentes, lo que implica un desafió para todos los médicos que laboran en los servicios de urgencias.

Con la publicación de este caso, el autor intenta alertar a la comunidad medica nacional acerca de una complicación infrecuente y poco conocida del uso de la cocaína y ampliar las causas ya conocidas de los casos de neumotórax "espontáneos".

\section{Caso Clínico}

El paciente es un varón de 24 años de edad, soltero, trabajador bananero y residente en $B$ ananito (zona rural de Limón), sin antecedentes médicos de importancia, tabaquismo desde los 17 años de edad (15 cigarrillos por día), consume en forma diaria marihuana y en algunas ocasiones la mezcla con Crack, inhala cocaína en forma muy ocasional (cuando tiene dinero) siendo la penúl tima vez hace año y medio.

Dos horas después de haber inhalado cocaína consulta al servicio de urgencias por dolor toráxico izquierdo de inicio súbito, el cual se incrementa con la inspiración y asociado con disnea progresiva. Al examen físico de ingreso tensión arterial de 117/84, pulso 96 por minuto, con disnea en reposo, tráquea desviada a la derecha y con hipoventilación basal izquierda. 
Se toma Radiografía de tórax (Fig. 1) la cual evidencia un neumotórax del $95 \%$, con desviación del mediastino hacia la derecha.

Se colocó un tubo de tórax izquierdo, en el quinto espacio intercostal línea medio axilar ( Fig.2) obteniéndose resolución completa del neumotórax, se hospitaliza para seguimiento y el tubo de tórax se retiró al cuarto día de su ingreso.

El paciente fue controlado en Consulta Externa de Cirugía General a los 9 y 30 días encontrándose asintomático.

\section{Discusión}

El mecanismo de acción de la cocaína como anestésico local es debido a su capacidad de bloquear la iniciación y conducción del impulso nervioso, al impedir la despolarización neuronal. Sus efectos sistémicos son debidos a un aumento en la actividad simpática, dado que impide la recaptación presináptica de dopamina, noradrenalina y adrenalina. ${ }^{1}$

L a cocaína es degradada por las colinesterasas plasmáticas y hepáticas en metabolitos hidrosolubles, los cuales se eliminan por la orina. Una baja actividad de la colinesterasa plasmática hace a los pacientes al tamente sensibles a pequeñas dosis de la droga. ${ }^{1}$

Desde 1982 empiezan a aparecer reportes en la literatura médica de infartos agudos del miocardio estrechamente relacionados con el uso de la cocaína ${ }^{2}$, además de reportes de arritmias tales como taquicardia sinusal, fibrilación ventricular y aun asistolias. ${ }^{3}$

También están descritos casos de isquemia intestinal secundarios al uso de cocaína, que han ameritado cirugía de urgencias puesto que han evolucionado hacia la perforación de víscera hueca ${ }^{5,6,7}$. En el cuadro, se resumen las posibles complicaciones asociados con el uso de la cocaína.

Las complicaciones pulmonares por el uso de la cocaína son debidas a barotrauma 9,10,11 aunque al gunos piensan que la cocaína podría producir toxicidad alveolar directa ${ }^{10}$. Al inhalar vigorosamente y realizar maniobras de Valsalva en forma fre cuente se produce un aumento de presión en la vía aérea el cual genera ruptura alveolar y escape aéreo, el que puede seguir varias rutas: hacia el espacio pleural ,al pericardio, al mediastino 0 a pared torácica, generando de esta manera los diversos cuadros clínicos asociados.

La fisiopatología del neumotórax hipertensivo se explica por un mecanismo de válvula unidi reccional que permite la entrada del aire al espacio pleural durante la inspiración y atrapamiento del mismo en la expiración, resultando así una acumulación progresiva y continua del aire en el espacio pleural que puede ser letal si no es tratado oportunamente. ${ }^{12}$

Shesser y colaboradores 10 , en el centro medico de la universidad George Washington, reportan dos pacientes femeninas que presentaron barotrauma secundario al uso de cocaína, las

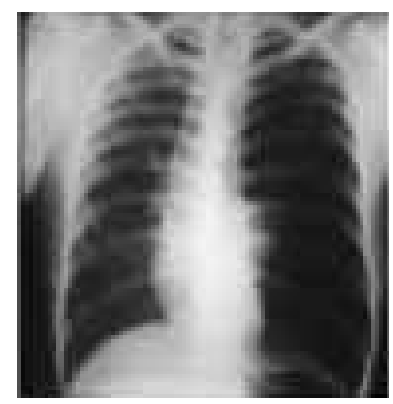

Figura 1: Neumotórax hipertensivo izquierdo con desplazamiento mediastinal contralateral.

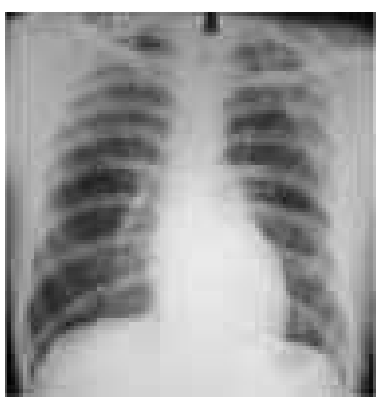

Figura 2: Tubo de tórax izquierdo con resolución completa del neumotórax. cuales consultaron al servicio de urgencias por dolor torácico sin disnea, en ambas se documentó neumomediastino y en una de ellas había neumotórax bilateral. La primera de ellas, de 26 años de edad había fumado cocaína durante seis horas en forma continua. La otra paciente de 19 años había inhalado cocaína una hora y media antes de iniciarse los síntomas. Las dos pacientes evolucionaron satisfactoriamente. No fue necesario colocar tubos de tórax en la paciente con neumotorax bilateral puesto que no tenia dificultad respiratoria.

EI Dr. A drouny y colaboradores ${ }^{11}$, en el $\mathrm{K}$ aiser Permanente $M$ edical Center en los A ngeles California, reporta el caso de un hombre de 20 años de edad quien presentó dolor retroesternal y cervical de inicio súbito asociado con disnea y enfisema subcutáneo en cuello junto con crepito mediastinal (signo de Hamman). Este paciente había inhalado crack a presión positiva ( después de inhalar la droga, su novia le había soplado boca a boca con la intención de aumentar los efectos de la cocaína), se le documentó neumomediastino y neumopericardio los cuales desaparecieron al dejar al paciente en reposo, el control clínico a los cinco meses mostró paciente asintomático.

El neumotórax "espontáneo" se define como la acumulación de aire en la cavidad pleural sin una causa aparente, casi siempre debido a rotura de un quiste, ampolla, 0 bulas subpleurales en un adulto joven, por lo común un varón fumador de cigarrillos ${ }^{12}$. Su tratamiento depende básicamente de dos factores: a) grado del colapso pulmonar y b) condición ventilatoria del paciente, así neumotórax menores del $25 \%$ con paciente asintomático se manejan conservadoramente sin colocar tubo de tórax, pero los pacientes con neumotórax mayores del $25 \%$ ameritan toracostomía cerrada (inserción de tubo de tórax) independientemente de los síntomas, también se recomienda colocarlo en pacientes con neumotórax menores del $25 \%$ sintomáticos o que van a ser sometidos a ventilación con presión positiva (cirugías con anestesia general y ventilación mecánica). ${ }^{13}$

Nuestro paciente presentaba un cuadro clínico típico de neumotórax hipertensivo, razón por la cual fue manejado con toracostomía cerrada (tubo de tórax) el cual fue colocado en el 
quinto espacio intercostal izquierdo a nivel de la línea axilar media, su evolución clínica fue satisfactoria con resolución completa del neumotórax.

Teniendo en cuenta el presente caso clínico y a los reportes de la literatura medica consultada ${ }^{9,10,11}$, se hace indispensable interrogar específicamente acerca del uso de cocaína en los pacientes que presentan un cuadro de neumotórax "espontáneo".

\begin{tabular}{|c|c|}
\hline Cardiacas $^{2.3}$ & $\begin{array}{l}\text { Infarto agudo del miocardio, } \\
\text { arritmias, cardiomiopatías. }\end{array}$ \\
\hline Neurológicas ${ }^{4}$ & $\begin{array}{l}\text { A ccidente cerebrovasculares, } \\
\text { convulsiones, psicosis paranoicas. }\end{array}$ \\
\hline Gastrointestinal es ${ }^{5.6 .7^{\prime}}$ & $\begin{array}{l}\text { Desprendimiento prematuro de } \\
\text { placenta, retardo del crecimiento } \\
\text { intrauterino. }\end{array}$ \\
\hline Pulmonares & $\begin{array}{l}\text { Edema agudo de pulmón, } \\
\text { neumomediastino, } \\
\text { neumopericardio, neumotórax. }\end{array}$ \\
\hline Otras ${ }^{1}$ & $\begin{array}{l}\text { Rabdomiolisis, perforación del } \\
\text { tabique nasal etc. }\end{array}$ \\
\hline
\end{tabular}

\section{Abstract}

The use of cocaine causes pulmonary, cardiac, obstetric, muskuloskeletal and gastrointestinal complications.

The case of a young man who sustained barotrauma (hipertensive pneumotorax) associated with the use of cocaine is presented here.

Based on this case and a review of the literature it is recommended that a careful drug abuse history be obtained when "spontaneous" pneumotorax is encountered in an adult or adolescent patient.

\section{Referencias}

1. Cregler. LL, M ark H. M edical complications of cocaine abuse. N Engl J M ed 1986; 315: 1495-1499

2. Cregler $L L, M$ ark $H$. Relation of acute myocardial infarction to cocaine abuse. A m J Cardiol 1985; 56: 794.

3. Benchimol $A, B$ artall $H$, Desser $K B$. A ccelerated ventricular rhythm and cocaine abuse. A nn Intern M ed 1978; 88: 519-520.

4. Schwartz KA, Cohen JA. Subarachnoid hemorrhage precipitated by cocaine snorting. A rch N eurol 1984; 41: 705.

5. Lee HS, LaM aute HR, Pizzi WF, et al: A cute gastroduodenal perforations associated with use of crack. A nn Surg 1990; 211: 15-17.

6. Muñiz $A E$, Evans T. A cute gastrointestinal manifestations associated with use of crack. A m J Emerg M ed 2001;19: 61-63.

7. Feliciano DV, Ojukwu JC, Rozycki GS, et al: The epidemic of cocainerelated juxtapyloric perforations: with a comment on the importance of testing for Helicobacter pylori. A nn Surg 1999; 229: 801-806.
8. Acker D, Sachs BP, Tracey KJ, Wise WE. A bruptio placentae associated with cocaine use. A m J Obstet Gynecol 1983; 146: 220-221.

9. Allred RJ, Ewer S. Fatal pulmonary edema following intravenous "freebase" cocaine use. A nn Emerg M ed 1981; 10: 441-442.

10. Shesser R, Davis C, Edelstein S. Pneumomediastinum and pneumothorax after inhaling alkaloidal cocaine. A nn Emerg M ed 1981; 10: 213215.

11. Adrouny A, Magnusson P. Pneumopericardium from cocaine inhalation. N Engl J Med 1985; 313: 48-49.

12. Takaro T, Scott SM. Pleura y empiema. En: D.C. Sabiston Tratado de patología quirúrgica. M éxico; Interamericana-M cG raw-Hill,1988.

13. Echavarria H, Ferrada R, K estenberg A . U rgencia Quirúrgica. Universidad del Valle: Cali, Colombia . M arzo 1988. 